\title{
Das internationale Autorenliedfestival "Lehesaju Muusika" in Tartu als spezifische Form der Kulturvermittlung und Analyseobjekt für Mehrsprachigkeitsforschung
}

\author{
ANASTASIA SHAKHOVA
}

\begin{abstract}
The Lehesaju Muusika International Music and Poetry Festival in Tartu as a Specific Form of Cultural Mediation and Object of Analysis for Research on Multilingualism. The paper focuses on the multilingual discourse of the Lehesaju Muusika international music and poetry festival, which takes place annually in Tartu, Estonia. Being an international cultural event organised by ethnic minorities, Lehesaju Muusika represents a unique source of empirical data for research on multilingualism. The festival attracts songwriters and performers of the so-called 'author song' or 'bard song' not only from Estonia, but also from all over the world. The key feature of this genre is the dominance of the text over the music.

The spatial organisation of a concert hall represents a specific power constellation within a microsocial structure. Performing artists have the power to decide in which language they perform and address the multilingual audience, while the audience itself has an indirect effect on this decision. The artist's dialogue with the audience represents a peculiar discursive entity within the discourse of the festival. Code-switching appears to be one of the inherent characteristics of this discursive entity.

The present paper summarises some key features of international music and poetry festivals as multilingual cultural events, focusing on the discourse of the Lehesaju Muusika festival. It offers a brief analysis of the audience's language profile based on the results of a microsociological case study carried out during the latest festival, in 2019. To illustrate the complexity of the multilingual communication during the festival, three situations of code-switching during the performance of an Estonian native speaker in front of the multilingual audience are described and analysed.
\end{abstract}

Keywords: multilingualism; code-switching; discourse; “author song”; microsociolinguistics 


\section{Einleitung}

Im Mittelpunkt der vorliegenden Fallstudie steht eine besondere Form der kulturellen Veranstaltung, die des Autorenliedfestivals „Lehesaju Muusika“, das für ein mehrsprachiges Publikum in Tartu, Estland organisiert wird und ein facettenreiches Untersuchungsobjekt für die Mehrsprachigkeitsforschung darstellt. Die sprachliche und kulturelle Situation im heutigen Estland macht das Land zu einem vielseitigen Untersuchungsobjekt im Bereich der Mehrsprachigkeitsforschung. „Estland unterscheidet sich hinsichtlich seiner ethnodemographischen und rechtlichen Situation erheblich vom Rest Ostmitteleuropas, was den hohen Grad an wissenschaftlicher Aufmerksamkeit erklärt, die das Land bei Politikwissenschaftlern, Soziologen und Sprachpolitikern erregt hat", merkt Verschik zutreffend an (Verschik 2005 : 305, Übersetzung der Verfasserin). Den Ergebnissen der letzten Volks- und Wohnungszählung (Population and housing censuses in Estonia, Latvia and Lithuania) zufolge gibt es 192 ethnische Gruppen in Estland, wobei die Bevölkerungszahl der meisten größeren ethnischen Gruppen im Vergleich mit der letzten Volkszählung zurückgegangen und die Anzahl der Ethnien im Allgemeinen gestiegen ist (siehe Schneider 2013).

Das kritische Umdenken hinsichtlich der längere Zeit geltenden Annahme, dass Monolingualismus der Normfall ist und dass Mehrsprachigkeit einen Sonderfall bzw. eine Abweichung von der Norm darstellt, sowie „die Tendenz, Sprachen nicht mehr als voneinander klar abgrenzbare Einheiten zu sehen“, sind Anzeichen eines Paradigmenwechsels in der Mehrsprachigkeitsforschung (Busch 2017: 81). Busch spricht von einem Paradigmenwechsel im Kuhn'schen Sinne, wobei sie auf den normativen Charakter eines Paradigmas und der sogenannten „normalen Wissenschaft“ aufmerksam macht (ebenda). An dieser Stelle würde sich lohnen, auch auf einen (potenziell) krisenhaften Charakter jeglichen Paradigmenwechsels hinzudeuten, selbst wenn dieser nicht explizit sichtbar wird. Kuhn zufolge entwickeln sich neue Theorien „nachdem eine normale Problemlösungstätigkeit offensichtlich versagt hatte“ (Kuhn 1978: 87). Dabei kann ein Paradigmenwechsel erst dann stattfinden, wenn das neue Paradigma stark genug ist, um das alte Paradigma abzulösen (siehe Kuhn 1978: 90).

Der notwendige Paradigmenwechsel wurde bereits Anfang des 21. Jahrhunderts in mehreren Publikationen vorausgesagt. Verschik wies bereits 2005 auf die Spannung hin, die aufgrund der Dominanz makrosoziologischer Ansätze in der Mehrsprachigkeitsforschung in Estland und wegen des Fehlens

1 https://www.stat.ee/sites/default/files/2020-08/2011\%20Population\%20and\%20 Housing\%20Censuses\%20in\%20Estonia\%2C\%20Latvia\%20and\%20Lithuania.pdf (30.10.20). 
ausreichender mikrosoziologischer Fallstudien der linguistischen Kreativität, Mechanismen und Praktiken der multilingualen Kommunikation entstanden ist (siehe Verschik $2005^{2}: 378$ ).

Der vorliegende Beitrag hat zum Zweck, durch eine Fallstudie des mehrsprachigen Diskurses des Autorenliedfestivals „Lehesaju Muusika“ (Tartu, Estland) die Perspektiven der mikrosoziologisch orientierten Mehrsprachigkeitsforschung mithilfe ethnographischer Methoden zu skizzieren. Eine besondere Aufmerksamkeit wird dabei dem Code Switching sowie den translationsbezogenen Aspekten der mehrsprachigen Kommunikation geschenkt. Hiermit soll auch die Annahme untermauert werden, dass mikrosoziologische Untersuchungen von Code Switching mit Schwerpunkt auf der sprachlichen Kreativität der SprecherInnen im konkreten Kulturkontakt (in der Performance-Situation) zum allgemeinen Bild der Mehrsprachigkeit beitragen und die Lücke zwischen dem traditionellen grammatischen und dem makrolinguistischen Ansatz in der Mehrsprachigkeitsforschung füllen können (siehe Verschick $2005^{2}: 381$ ).

\section{Das Autorenliedfestival „Lehesaju Muusika“ als spezifische Form der Kulturvermittlung: Besonderheiten, Anregungen, Perspektiven}

\subsection{Das Autorenlied als Genre: Einblick in die Geschichte und aktueller Stand}

Das Genre des sowjetrussischen Autorenliedes entwickelte sich als ein spezifisches kulturelles Phänomen in den 1960er Jahren während der sogenannten Tauwetterperiode in der sowjetischen Politik und verbreitete sich schnell unter der sowjetischen Intelligentzija (Orlova 2011: 120). Das Autorenlied wird als ein von einer Person selbst geschaffenes bzw. komponiertes sowie selbst vorgetragenes und selbst begleitetes Lied definiert ${ }^{2}$ (siehe Orlova 2011: 121). Es kann

2 Zur Begriffsbestimmung des Autorenlieds in den deutschsprachigen Diskursen gibt es die 2010 veröffentlichte Monographie von S. Hammer, der das Autorenlied als „ein von einer Person vollständig selbst geschaffenes sowie solistisch selbst vorgetragenes und instrumental selbst begleitetes Lied“ definiert (Hammer 2010: 56). Unter, AutorenliedKunst' versteht Hammer "alles von einem oder mehreren Liedermacher(n) während einer Darbietung auf der Bühne Aufgeführte und alle Elemente dieser Aufführung (also inkl. Publikumsdialog u.a.)" (Hammer 2010: 56). Im Mittelpunkt der Publikation von Hammer stehen die Lieder von Mani Matters. Bei seiner Definition orientiert sich Hammer an der Form sowie an der Darstellungsform, obwohl er selber in einem früheren Kapitel seines Werkes auf die inhaltlichen Besonderheiten aufmerksam macht. Dazu gehören der politische bzw. sozialkritische Aspekt (Hammer 2010: 4041), sowie der nicht-kommerzielle Charakter des Autorenliedes. 
vorkommen, dass ein Lied aus der Zusammenarbeit mehrerer Liedermacher resultiert und somit auch mehrere Autoren hat.

Die dominierende Rolle wird dabei dem Text zugewiesen, er beeinflusst die musikalischen Aspekte des Autorenliedes sowie das Darstellungsformat (Orlova 2011: 121). Zu den bekanntesten Vertretern des Genres zählen Bulat Okudžava, Jurij Visbor, Alexandr Galič, Novella Matveeva, Vladimir Vysockij $^{3}$ u.a.. Orlova betont, dass die Persönlichkeits- und Meinungsfreiheit sowie die Idee einer freien Schöpfung eine zentrale Rolle in den Werken der sowjetischen „Barden“ eingenommen haben, was zur Zensur ihrer Werke unter den politischen Umständen einer fortlaufenden staatlichen Kontrolle des Kulturlebens bis hin zur Verfolgung einzelner Autoren geführt hat (Orlova 2011: 121).

Der sozialkritische Charakter und die politische Bedeutung des Autorenliedes zu dem Zeitpunkt seines Aufblühens werden auch in den Publikationen zeitgenössischer Sozial- und Kulturforscher hervorgehoben. „Der Beitrag der Bardenkultur erschöpft sich jedoch nicht allein im Politischen“, so Poljakov (2011: 115). Im Mittelpunkt eines Autorenliedes stand oft die geistige Welt eines Menschen, seine Gedanken und Gefühle, wobei diese Themen der damaligen sowjetischen Unterhaltungsmusik fremd waren (siehe Poljakov 2011: 115). Dank ihrer Thematik bleiben Vysockij- und Okudžava-Lieder auch heutzutage relevant und aktuell.

Olson analysiert die musikalischen Wurzeln des sowjetrussischen Autorenliedes und kommt zur Schlussfolgerung, dass es seinen Ursprung in Gaunerlied und Bänkellied nimmt. Dadurch, dass Autorenlieder von Amateuren (nichtAutoren) auswendig gelernt und als mündliches Kulturgut vorgetragen werden, weist das Autorenlied gewisse Ähnlichkeiten mit Volksliedern auf (Olson 2004: 72). Der musikalischen Komponente des Autorenliedes wird eine sekundäre Rolle zugewiesen, daher ist der laienhafte Aspekt der Darbietung zu einem Gattungsmerkmal geworden: Eine ungeübte Sängerstimme und eine nicht-professionelle musikalische Selbstbegleitung (Gitarre) unterscheiden das Autorenlied von der Unterhaltungsmusik (Olson 2004: 72).

Das Genre des Autorenlieds ist in den russischsprachigen Gemeinschaften in Estland, Lettland, Deutschland, Frankreich und in der Tschechischen Republik $^{4}$ beliebt. Ein Autorenliedfestival hat zum Zweck, Lieder beliebter Vertreter des Genres zu verbreiten beziehungsweise zu pflegen und vor dem

\footnotetext{
Siehe dazu Boss, D. 1985. Das sowjetrussische Autorenlied: eine Untersuchung am Beispiel des Schaffens von Aleksandr Galič, Bulat Okudžava und Vladimir Vysockij. München: Otto Sagner.

4 In diesen Ländern finden regelmäßig Autorenliedfestivals statt.
} 
Vergessen zu bewahren sowie das Fortleben des Genres durch die Werke zeitgenössischer jüngerer Autoren zu sichern. Als Form der Selbstdarstellung verlangt das Autorenlied dabei keine nennenswerte Musikausbildung, die meisten Lieder können, wie bereits erwähnt, mit geringen Gitarrenkenntnissen erlernt und vorgetragen werden.

\subsection{Autorenliedfestivals in Estland: „Lehesaju Muusika“}

\subsection{1 „Lehesaju Muusika“ als Kulturphänomen}

In Estland finden regelmäßig Autorenliedfestivals statt. Im Folgenden werden einige Festivals genannt, die bereits auf eine langjährige Geschichte zurückblicken:

- „Balti Keeled“/„Baltische Sprachen“. Der russischsprachige Name lautet „Baltijskije struny“ (etwa „Saiten des Baltikums“). ${ }^{5}$ Das Festival findet jährlich in Narva Jõesuu statt.

- „Lehesaju Muusika“/„Laubfall-Musik“/„Muzyka listopada“. Das Festival findet jährlich in Tartu statt. „Lehesaju Muusika“ wurde vom Anfang an als Autorenliedfestival konzipiert. Im Laufe seiner mehr als zehnjährigen Geschichte entwickelte sich das Festival zu einem Musik- und Poesiefestival, in dessen Rahmen mehrere Genres und Stile vertreten sind. Dennoch wird das Festival in lokalen Medien als „autorilaulu festival“6 (Autorenliedfestival) oder „rahvusvaheline autorilaulu festi-

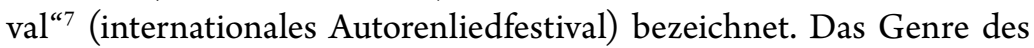
Autorenliedes dominiert allerdings auf der Bühne. Über das Festival berichten sowohl estnischsprachige als auch russischsprachige Medien. In den Artikeln werden die Internationalität und die Mehrsprachigkeit des Festivals sowie seine Rolle für das Kulturleben Tartus betont.

- „Svet v dekabre“/„Licht im Dezember“. Das Festival verdankt seinen Namen dem Poesie-Sammelband des russisch-estnischen Dichters Svetlan Semenenko, der unter anderem als Übersetzer von Jaan Kaplinski bekannt ist. Das Festival findet in Tallinn im Dezember statt.

Die Musikkultur der russischsprachigen Migranten unterscheidet sich von dem heutigen Entwicklungsstand des Autorenliedes in Russland. In Estland gibt es

https://www.balticstrings.com/ (30.10.20).

6 Siehe auf Estnisch https://www.vooremaa.ee/nadalavahetusel-taidab-tartu-lehesajumuusika/; https://www.vooremaa.ee/nadalavahetusel-taidab-tartu-lehesajumuusika/.

7 https://blogi.kultuur.info/festivalide-kalender-2019/ (30.10.20). 
SHAKHOVA

außerdem keine vergleichbare Tradition des Autorenliedes in dem Sinne, in dem der Terminus "Autorenlied" in russischsprachigen Kreisen verwendet wird. Daher sollte die Veranstaltung eines Autorenliedfestivals in Estland, an dem estnischsprachige Autoren neben russischsprachigen Autoren auftreten und selbstgemachte Lieder auf Estnisch vortragen, nicht nur als eine kulturelle Interferenz (siehe Even-Zohar 2008: 117-118) betrachtet werden, sondern als der Ursprung eines qualitativ neuen Genres, welches sowohl die Merkmale des Autorenliedes besitzt als auch bestimmte Merkmale des estnischen Volksliedes, der modernen estnischen Lyrik und der westlichen Musiktradition (Blues, Jazz) aufweist.

Das „Lehesaju Muusika“-Festival unterscheidet sich in vielen Aspekten von den restlichen Autorenliedfestivals, die in Estland und in anderen Ländern stattfinden. Im Rahmen dieses Festivals findet ein Wettbewerb von Autoren und Interpreten statt. Der Preisträger wird durch die Abstimmung des Publikums ermittelt. Dabei bemühen sich die Organisatoren darum, estnische Autoren zu engagieren und sie zur Teilnahme am Wettbewerb zu motivieren. Estnischsprachige Autoren dürfen jedes Jahr am Liedermacherwettbewerb teilnehmen, die Autoren aus anderen Ländern dürfen hingegen nur zweimal auftreten. Diese Tatsache lässt sich durch Patronage (in der Lefevere'schen Terminologie ${ }^{8}$ ) erklären, die die Organisatoren ausüben, um das Festival attraktiv für estnischsprachige Zuschauer zu machen. Die Organisatoren legen großen Wert darauf, dass estnische Autoren ihre Lieder auf Estnisch vortragen. Auch dieses Jahr wurde das Konzert mit dem Auftritt estnischer Autoren eröffnet. 2018 wurde der Hauptpreis des Festivals dem estnischen Künstler und Liedermacher Silver Sepp verliehen. Dieses Jahr trat er wieder auf neben dem Familienensemble Die Johansons (est. Johansonide pereansambel), Priit Strandberg und Tõnis Mägi, wobei letzterer als prominenter Gast am Festival teilgenommen hat.

Das Festival genießt eine intensive staatliche Förderung, was der Organisatorin Ljudmilla Mesropjan und ihrer aktiven Zusammenarbeit mit der Stadtverwaltung zu verdanken ist. Beim Festival 2019 war auch der Bürgermeister von Tartu, Urmas Klaas, anwesend.

Darüber hinaus versuchen die Veranstalter nicht nur Liedermacher und Interpreten, sondern auch Maler einzuladen, die ihre Gemälde im Rahmen des Festivals vorstellen. ${ }^{9}$ „Lehesaju Muusika“ ist das einzige Autorenlied-Festival,

8 Lefevere, A. 2009. Interpretation, Übersetzung, Neuschreibung: Ein alternatives Paradigma. - S. Hagemann, ed., Deskriptive Übersetzungsforschung. Eine Auswahl. Translationswissenschaftliche Bibliothek 4. Berlin: Saxa, 63-92. Ins Deutsche übersetzt von Susanne Hagemann.

9 Siehe https://www.erm.ee/et/events/lehesaju-muusika (30.10.20). 
das sich multimedial präsentiert. Dieses Jahr fanden die Ausstellungen sowie das Jubiläumskonzert im Estnischen Nationalmuseum Tartu (ERM) statt.

\subsubsection{Der mehrsprachige Diskurs des „Lehesaju Muusika“-Festivals als sprachlich, sozial und diskursiv gestalteter Raum}

Das Programm eines Autorenliedfestivals ist immer räumlich und zeitlich begrenzt. Konzerte auf der Bühne (oft auf einer improvisierten oder symbolischen Bühne) dauern mehrere Stunden, dabei treten mehrere Autoren und Interpreten auf. Während die Organisatoren entscheiden, wer und wie lange auf der Bühne auftreten darf, können die Liedermacher selber bestimmen, welche Lieder sie vortragen und in welcher Sprache. Dadurch, dass solche Konzerte meistens ein Thema oder ein Motto haben, werden einzelne Auftritte in der Regel thematisch verbunden. Die Organisatoren übernehmen auch die moderierende Rolle und begleiten die Auftritte mit ihren Kommentaren, dazu gehören auch die Begrüßung und das Schlusswort, eine Kurzfassung des Inhalts eines Liedes oder Anmerkungen zur auftretenden Person.

Während ihres Auftritts führen Liedermacher ab und $\mathrm{zu}$ selber einen Publikumsdialog, dennoch ist ein Auftritt an sich bereits eine Einladung zum Dialog: Er löst eine Reaktion des Publikums aus und setzt eine „Antwort“ voraus in Form von Applaus, Lachen, Schweigen, Weinen, Empörung oder Zustimmung des Publikums usw. Der performative Charakter dieser Handlung macht die während eines Liedermacherfestivals stattfindenden Konzerte mit Theateraufführungen vergleichbar. Dabei nehmen die Zuschauer ein Konzert auch als Gesamtheit wahr, also wie eine Aufführung. Dank der räumlichen und temporären Abgegrenztheit sowie der thematischen Verbundenheit lässt sich alles, was im Rahmen des Festivals auf der Bühne gesagt und gesungen wird, als interner Diskurs des Festivals bezeichnen. Zum Gesamtdiskurs des Festivals gehört auch das umfangreiche audiovisuelle Material: Werbung, Flyer, Plakate, Reportagen, Webseiteninhalte usw.

Die oben beschriebenen Eigenschaften des Autorenliedfestivals „Lehesaju Muusika“ sprechen dafür, das Festival als einen symbolischen sprachlich und sozial konstruierten diskursiven Raum zu betrachten, der seine Realisierung einem konkreten Raum, nämlich dem Konzertraum, findet (vgl. Busch 2017: 126). Der reale Raum veranschaulicht die komplexen Machstrukturen im mehrsprachigen Diskurs des symbolischen Raums sowie die Mechanismen der Machtverteilung und Kontrolle der Diskursproduktion. „Kleinräumige Sprachregime“ sind „durch spezifische Machtkonstellationen und sprachliche Hierarchien gekennzeichnet wie durch gegenläufige Aneignungsprozesse oder Gegendiskurse, die geeignet sind, sprachliche Praktiken und Sprachideologien zu transformieren“ (Busch 2017: 126). 
SHAKHOVA

Die Bühne, auf welcher nur die von den Organisatoren zugelassenen Liedermacher auftreten dürfen, zieht eine deutliche Grenze zwischen dem Publikum als Gesamtheit und den auftretenden Künstlern. Das Mikrophon stellt dabei ein sichtbares Machtsymbol dar: Es verleiht den Sprechenden oder den Singenden die Macht, den Diskurs des Festivals nach ihrem Wunsch zu gestalten (dazu gehört auch die Sprachwahl der „Bühnensprache“). Die „unsichtbare“ verborgene Macht gehört den Organisatoren: Sie entscheiden, wie lange und in welcher Reihenfolge die Liedermacher auftreten dürfen.

Das „Lehesaju Muusika“-Festival bietet facettenreiche Möglichkeiten für die ethnografische Forschung im Bereich der Mehrsprachigkeit: Es ermöglicht eine teilnehmende Beobachtung, die Analyse von Ton- und Bildaufnahmen, Umfragen und qualitative Interviews (siehe auch Busch 2017: 149).

Bühnenauftritte bzw. ganze Konzerte inklusive der Moderation werden regelmäßig aufgenommen, das umfangreiche Video- und Audiomaterial samt Fotografien, Postern usw. wird sorgfältig aufbewahrt und zum Teil veröffentlicht.

\section{Ergebnisse der Fallstudie}

3.1 Die Sprachen des Festivals „Lehesaju Mussika“: Eine Skizze des Publikumsprofils

Das Festival „Lehesaju Muusika“ lockt viele mehrsprachige Teilnehmer an, die literarische Werke (Gedichte, Liedertexte) in mehreren Sprachen verfassen und in mehreren Sprachen vortragen. Laut den Ergebnissen einer Umfrage, ${ }^{10}$ die ich während des zehnten Jubiläumsfestivals 2019 durchführen durfte, werden mindestens siebzehn Sprachen von den TeilnehmerInnen des Festivals vertreten, darunter Estnisch, Russisch, Ukrainisch, Lettisch, Finnisch, Armenisch, Wiesenmari (wolgafinnische Gruppe) und Deutsch. Diese Sprachen wurden als (eine der) Muttersprachen angezeigt. Als weitere Sprachen wurden Georgisch, Englisch, Griechisch, Schwedisch, Französisch, Italienisch, Polnisch, Hebräisch und Jiddisch genannt.

Es wäre daher eine Vereinfachung, den Diskurs des Festivals als „zweisprachig“, Estnisch-Russisch oder Russisch-Estnisch zu betrachten, wobei die Rollen und Funktionen verschiedener Sprachen im Gesamtdiskurs des Festivals unterschiedlich sind. Estnisch und Russisch übernehmen im Rahmen des Diskurses des Festivals ähnliche und dennoch unterschiedliche Funktionen.

1047 Fragebögen wurden freiwillig von den TeilnehmerInnen des Festivals ausgefüllt und unterschrieben, die Ergebnisse wurden qualitativ und quantitativ ausgewertet. 
Beide Sprachen sind sozusagen Bühnensprachen, beide werden zwecks Ankündigungen und Werbung verwendet, sowie in der Kommunikation mit Behörden. Die zwischenmenschliche Kommunikation im Rahmen des Festivals ist keinesfalls nur auf Estnisch und Russisch beschränkt.

Ca. 34\% der Befragten bezeichnen sich als zwei- oder mehrsprachig. Fast alle BefragungsteilnehmerInnen, die mehr als eine Erstsprache aufgeführt haben, verfassen literarische Werke (Gedichte, Liedtexte) in mehreren Sprachen. Es handelt sich dabei vor allem, aber nicht ausschließlich, um ihre Erstsprache(n). Beispielsweise verfasst und veröffentlicht eine Befragungsteilnehmerin (L1 Russisch) literarische Werke auf Estnisch und Deutsch, ein anderer Befragungsteilnehmer (Erstsprachen Russisch und Deutsch) verfasst und veröffentlicht literarische Werke auf Englisch, Französisch, Jiddisch und Ukrainisch ${ }^{11}$. Nur wenige BefragungsteilnehmerInnen, die sich als zweisprachig (aufgewachsen) identifizieren, verfassen keine literarischen Werke.

Ca. 15\% der Befragten haben sich als ausschließlich einsprachig identifiziert (L1 Russisch). Rund 51\% der Befragten haben angegeben, dass sie mehr als eine Sprache beherrschen, haben sich allerdings nicht als bilingual bezeichnet.

Bemerkenswert ist die folgende Tatsache: Fast alle Befragten haben angezeigt, dass sie Englisch als erste oder zweite Fremdsprache beherrschen. Allerdings wird Englisch nur im Ausnahmefall auf der Bühne gesprochen (siehe Situationsanalyse im nächsten Kapitel des vorliegenden Beitrages).

Während des Konzerts und in den Pausen werden Russisch (öfter) und Estnisch (seltener) zur lingua franca in der Kommunikation zwischen den Vertretern unterschiedlicher Kultur- und Sprachgemeinschaften. Wenn die Vertreter derselben Gemeinschaft kommunizieren, bevorzugen sie die Sprache der Gemeinschaft (z.B. Armenisch).

Die Auflistung der Sprachen, die den Diskurs des Festivals mitgestalten, stellt nur die Spitze des Eisbergs dar. Der Diskurs des Festivals veranschaulicht die Annahme, dass es keine klar voneinander abgrenzbaren zählbaren Sprachen gibt: Das gesprochene Estnisch unterscheidet sich von der Sprache der estnischen Lyrik, die von der Bühne zu hören ist, sowie von der Fachsprache der professionellen Musiker und Tontechniker.

\subsection{Sprachbiographie: Ljudmilla Mesropjan}

Das Konzept des internationalen Autorenliedfestivals „Lehesaju Muusika“ wurde ursprünglich von Ljudmilla Mesropjan entworfen und realisiert. Für ihren wertvollen Beitrag zum kulturellen Leben von Tartu und unter anderem

11 Selbsbewertung 
für die Veranstaltung des „Lehesaju Muusika“-Festivals erhielt Mesropjan 2020 am Stadtfest eine Auszeichnung der Stadt. ${ }^{12}$

Mesropjans eigene Sprachbiographie stellt ein markantes Beispiel dafür dar, welche Rolle die Mehrsprachigkeit im kulturellen Leben ethnischer Minderheiten in Estland spielt. Mit einer skizzenhaften Beschreibung ihres Lebenslaufs möchte ich die Wichtigkeit mikrosoziologischer Fallstudien in der Mehrsprachigkeitsforschung erneut hervorheben.

Die Hauptorganisatorin des Festivals Ljudmilla Mesropjan ist im Jahre 1960 in Tschetschenien in einer armenischen Familie geboren. Zuhause wurden Armenisch und Russisch gesprochen, während Mesropjan als Kind noch Ingusch passiv beherrschte. Als sie dreizehn Jahre alt war, zog ihre Familie in die Stawropol Region, wo Russisch zur alltäglichen Kommunikationssprache wurde. Nach einem Aufenthalt in St. Petersburg zog die 21-jährige Mesropjan 1981 mit ihrem Mann nach Eriwan. Dort erlebte sie zum ersten Mal das literarische Armenisch, eine Sprache, die sich von dem Armenischen, das in der Familie gesprochen wurde, unterschied, und in die sie sich noch einarbeiten musste. Russisch verwendete Mesropjan jedoch in den Fällen, in denen ihr eigenes Sprachrepertoire (Armenisch) nicht ausreichend war.

Seit 1986 lebt Mesropjan in Estland. Somit fügte sich Estnisch zu ihren Kommunikationssprachen hinzu. Der Erwerb der estnischen Sprache wurde am Anfang ihres Aufenthaltes in Estland dadurch erschwert, dass die Hauptkommunikationssprachen in ihrer Gemeinschaft Armenisch und Russisch waren.

Aktuell wird Armenisch in der Familie gesprochen, das Russisch bleibt die Sprache der geistigen Tätigkeit, Estnisch ist zur Geschäftssprache geworden.

Einerseits veranschaulicht Mesropjans Sprachbiographie Verschiks These, dass die Vertreter von nicht-estnischsprachigen und nicht-russischsprachigen ethnischen Minderheiten in Estland sich durch geringe Sprachloyalität zur eigenen Erstsprache auszeichnen und in den meisten Fällen Russisch als Kommunikationssprache bevorzugen (Verschik 2005' 306 ). Andererseits wäre es eine Vereinfachung, die Verwendung des Russischen bei der Organisation des Festivals durch geringe Sprachloyalität zum Armenischen zu erklären: Mesropjans mündliche Kommunikation mit armenischsprachigen Künstlern und Bekannten erfolgt auf Armenisch, beim Schriftverkehr fällt die Entscheidung auf das Russische, da Mesropjan des armenischen Alphabets unkundig ist.

Verschik zufolge wird die Spezifik der mikrosoziolinguistischen Prozesse, die unter den Vertretern der ethnischen Minderheiten stattfinden, kaum

12 https://www.tartu.ee/et/tunnustatud-isikud/ljudmilla-mesropjan (30.10.20). 
untersucht (Verschik 2005ㄹ : 306). Da die Vertreter der armenischen Minderheit in Estland dank Mesropjans Mitwirkung regelmäßig am „Lehesaju Muusika"-Festival teilnehmen, sorgt das Festival für ein günstiges Umfeld für die mikrosoziologische Untersuchung der Mehrsprachigkeit unter den Armenisch-Muttersprachlern in Estland durch teilnehmende Beobachtung, qualitative Interviews und Analyse von Sprachbiographien.

4 Analyse von Ton- und Videoaufnahmen: Code Switching und Übersetzung

\subsection{Code Switching}

Während die Kommunikation zwischen den TeilnehmerInnenn und ZuschauerInnen des Festivals vor allem durch die teilnehmende Beobachtung untersucht werden kann, besteht für Bühnenauftritte die Möglichkeit einer Ton- und Videoaufnahmeanalyse, da die Konzerte des „Lehesaju Muusika“Festivals durch Videoaufnahmen sorgfältig dokumentiert werden. ${ }^{13} \mathrm{Im}$ vorliegenden Kapitel werden die Ergebnisse einer Videoaufnahmeanalyse von Bühnenauftrittsfragmenten mit Code Switching und Übersetzung vorgestellt.

Code Switching (CS) als Phänomen ist typisch für Kommunikationssituationen in Migrantengemeinschaften, bei regionalen Minderheiten und multilingualen Sprechergruppen (Gardner-Chloros 2009: 20). Verschik vertritt die Auffassung, dass CS in der langfristigen Perspektive zur Norm in einer mehrsprachigen Gemeinschaft sowie zum Symbol der neuen Identität werden kann (Verschik 2016: 5, vgl. auch Verschik 2004: 427). Bei Verschik wird CS als Oberbegriff für den Gebrauch mehrerer Sprachen in einer Konversation verwendet, um eine terminologische Verwirrung (code switching vs. code mixing) zu vermeiden (Verschik 2004: 428).

Im Rahmen der vorliegenden Fallstudie wird in Anlehnung an Auer (1998) die Annahme vertreten, dass der Kontrast zwischen zwei oder mehreren Codes im Code Switching bedeutungsgenerierend ist und entweder als Kontextualisierung einer Situation (diskursbezogenes CS) oder Hervorhebung bestimmter Merkmale des Kommunikationsteilnehmers (teilnehmerbezogenes CS) interpretiert werden kann (Auer 1998: 2).

Auer weist darauf hin, dass CS auch von KommunikationsteilnehmerInnen mit geringen Kenntnissen der „anderen“ Sprache initiiert werden kann und dass Bilingualismus keine unabdingbare Voraussetzung für CS ist (Auer 1998: 3).

13 Die Autorin des vorliegenden Beitrages dankt Vladimir Movschits für das zur Verfügung gestellte Videomaterial. 
Ein interessantes Beispiel dafür stellt der Auftritt ${ }^{14}$ von Silver Sepp während des Abschlusskonzerts des „Lehesaju Muusika“-Festivals 2019 vor dem mehrsprachigen Publikum dar (siehe die Publikumsanalyse in Kap. 3.1.)

Silver Sepp, ein estnischer Liedermacher aus Tartu und Estnisch-Muttersprachler, begleitet seine Auftritte mit selbstgemachten Musikinstrumenten. Sein Repertoire umfasst sowohl eigene Lieder als auch überarbeitete Versionen von den estnischen Regilaul ${ }^{15}$-Stücken. 2019 erhielt Silver Sepp den Publikumspreis und eröffnete das Abschlusskonzert des Festivals 2019. Das $\mathrm{zu}$ analysierende Videofragment befindet sich auf https://www.youtube. com/watch?v=aAFkd-WTuGQ. Als nächstes werden einzelne Fragmente des Auftritts von Silver Sepp analysiert.

\subsection{Situationen und Kommentare}

Situation 1. Silver Sepp beginnt seinen Auftritt (ohne Begrüßung) mit einer überarbeiteten Interpretation der Hymne des Festivals. Den ersten Vers singt er auf Russisch, der Text ist dem Publikum bereits bekannt (01:00-02:50). Der zweite Vers wird auf Estnisch vorgetragen ((1), 03:50-06:30). Während des Auftritts möchte Silver Sepp das Publikum zum Mitsingen motivieren. Er sagt auf Russisch ((2); 06:46): „Požalusta pomogi mne!“ (,bitte helfe mir!) und setzt danach den Auftritt auf Estnisch fort. ${ }^{16}$ Circa eine Minute später wiederholt Silver Sepp den Aufruf zum Mitsingen zuerst mit einer Geste und dann auf English ((3); 07:45-07:48): „Harmony... harmonize!“ (,Harmonie... harmonisiert!'). Die Aufführung geht weiter auf Estnisch und endet mit der Wiederholung einer Zeile der Hymne auf Russisch ((4); 09:25-09:32) und derselben Zeile auf Estnisch.

Kommentar zu Situation 1. Sepps Interpretation der Hymne des Festivals stellt eine symbolische Hybridisierung mehrerer Stile und Genres dar, sowohl aus der sprachlichen als auch aus der musikalischen Perspektive. Die russischsprachige Variante der Hymne entspricht den Besonderheiten des AutorenliedGenres (siehe 2.1). Durch die untypische Begleitung mit selbstgemachten Instrumenten, die Silver Sepp beim Vortragen seiner Lieder auf Estnisch

${ }^{14}$ Das vollständige Video siehe auf https://www.youtube.com/watch?v=aAFkdWTuGQ (30.10.20).

15 Estnische Runolieder (Volkslieder). Eine Textsammlung befindet unter den Link: http://regilaulik.folklore.ee/pohjatartumaa/louna-tartumaa/ilmast-ilma/luurilisedlaulud-maast-ja-ilmast/_(30.10.20).

16 07:28-07:30: Im estnischen Text kommt ausnahmsweise der Ausdruck „avtorskoj pesni“ vor („Autorenlieder“, GEN). 
verwendet, setzt er den Text bereits in einen anderen musikalischen Kontext. Das abwechselnde Vortragen auf Estnisch und auf Russisch (CS im Rahmen des Liedes) kann als ein künstlerisches Mittel betrachtet werden: Das Estnische und das Russische werden als Fragmente eines Ganzen dargestellt, als zwei Verse eines Liedes.

(2) und (3) zeichnen den Anfang eines Publikumsdialogs aus. Beide Äußerungen stellen ein Beispiel für CS dar und haben einen konkreten Zweck, nämlich, das Publikum zum Mitsingen zu animieren. Die Entscheidung fällt auf Russisch (1): Sepp als Estnisch-Muttersprachler empfindet das Publikum als „anderssprachig/russischsprachig“. Er weiß, dass das Publikum größtenteils aus den russischen Esten besteht und dass die Zuschauer sowohl Russisch als auch Estnisch sprechen. Im Laufe seines Auftritts fordert er das Publikum sogar mehrmals zum Mitsingen auf Estnisch auf.

Es lohnt sich in dieser Hinsicht, auf die Änderungstendenzen der Spezifik der sprachlichen und soziokulturellen Kontakte zwischen EstnischMuttersprachlern und den Vertretern sprachlicher Minderheiten aufmerksam zu machen: „Die Zunahme der Kompetenz in Estnisch als Zweitsprache unter Nicht-Esten hat Innovationen im (sozio-)sprachlichen Verhalten und im sprachlichen Repertoire ausgelöst, wobei CS möglicherweise eines der sichtbarsten und herausragendsten neuen Merkmale ist" ${ }^{17}$ (Verschik 2004: 427, Übers. der Verfasserin). Daher solle CS in Situation 1 (und auch in den folgenden Situationen 2 und 3) nicht bloß als Anzeichen einer möglichen Diskrepanz und Anerkennung der Anderssprachigkeit des Publikums interpretiert, sondern als ein verbindendes Kommunikationselement betrachtet werden, welches eine erfolgreiche Kommunikation sicherstellen soll.

Der spontane Wechsel zum Englischen (3) scheint durch die sprachbezogene (prosodische) Besonderheit der estnischen, russischen und englischen Benennungen für „Harmonie“ und „Harmonisieren“ (Verb) verursacht zu sein. Die Betonung fällt auf die erste Silbe bei harmony (Eng) und auf die zweite Silbe bei harmoonia (Est) oder garmonija (Rus). Somit passt das Englische besser zum rhythmischen Pattern in Silver Sepps Lied.

4.2.2 Situation 2. Silver Sepp beendet die Hymne des Festivals auf Estnisch und kündigt das zweite Lied an (11:17-11:55; Estnisch wird durch Fettdruck markiert, Russisch ist kursiv, Englisch wird unterstrichen):

17 Es muss an der Stelle jedoch auch Folgendes anerkannt werden: „Ein bedeutender Teil der gesellschaftlichen Struktur, einschließlich der Massenmedien, ist immer noch sprachlich zwischen Estnisch- und Russischsprachigen getrennt “ [...] (Vihalemm und Leppik 2019: 240, Übers. der Verfasserin). 
SHAKHOVA

1 Sledujušij ${ }^{18}$ pesnja eta očen stariy

,Das nächste Lied ist ein sehr altes Lied [...]

2 The main line of the song goes back to Estonian

,Die Hauptzeile des Liedes geht zurück auf die estnische

3 tradicii

tradicionalnyj pesni

,Tradition, traditionelle Lieder...

$4 \underline{\text { like thousands [of] years }}$

as a runo tradition

,wie die tausend Jahre [alte]

Runenliedertradition,'

5 as a Kalevala metric song

,wie ein metrisches Kalevala-Lied,

6 and the question in the song is

,und die Frage, die im Lied gestellt wird, lautet:

7 Kus (sic!) ${ }^{19}$ me’ läh'me vasta ööda, vasta ööda,

,Wohin gehen wir gegen die Nacht, gegen die Nacht,'

8 vasta kuuda, vasta pilgasta pimedad

'gegen den Mond, gegen die stockfinstere Dunkelheit'

8 how are we going through the night 9 kakmy [Pause]pošli

,Wie gehen wir durch die Nacht?'

$10[\ldots]$ läbi?

11 čerez noč

,Wie sind wir gegangen...

,durch“

'durch die Nacht!'

12 Jah!

$\mathrm{Ja}$ !

Kommentar zu Situation 2. Silver Sepp möchte sein nächstes Lied ankündigen, welches aus überarbeiteten Fragmenten mehrerer „regilaul“-Stücke besteht. Die Hymne des Festivals (der estnische Teil) wurde in modernem Estnisch vorgetragen, daher kann man den Übergang zum „regilaul“-Genre, selbst wenn es sich um eine Stilisierung handelt, bereits als CS betrachten. Silver Sepp möchte sein Publikum an diesen Übergang heranführen und fängt auf Russisch an über die Besonderheiten des nächsten Stücks zu erzählen (1), was an sich auch schon CS ist und den Anfang eines neuen diskursiven Kontinuums, nämlich des Publikumsdialogs, auszeichnet.

Die Entscheidung, das Publikum auf Russisch anzusprechen, erklärt sich wieder dadurch, dass Silver Sepp das Publikum als ,anderssprachig/russischsprachig" betrachtet. Der spontane Wechsel zum Englischen lässt sich zum Teil dadurch erklären, dass sein Repertoire auf Russisch unzureichend ist, dennoch entscheidet er sich nicht für Estnisch (L1). Das Fortsetzen des Publikumsdialogs auf English deutet auf Entgegenkommen, auf einen Kompromiss zwecks funktio-

\footnotetext{
${ }_{18}$ Hier Maskulinum.

19 Kuhu me läheme vastu ööd, vastu ööd, vast kuud, vastu pimedat (est).
} 
nierender Kommunikation hin: Keiner der Kommunikationsteilnehmenden spricht Englisch als Muttersprache, jedoch wird die Verständigung gewährleistet. Der Wechsel zum Russischen (3) ist wieder unerwartet und lässt sich dadurch erklären, dass dieser Ausdruck in Sepps russischsprachigem Repertoire vorhanden ist. Danach folgt der Wechsel zum Englischen (4). Die Sprechgeschwindigkeit wird dabei deutlich höher, eine bündige Beschreibung des „regilaul“-Genres auf Englisch scheint zum Teil der Performance geworden zu sein. Der Wechsel zum stilisierten Liedtext (7) wird durch die höchste Sprechgeschwindigkeit und ein neues rhythmisches Pattern gekennzeichnet. Der weitere Wechsel zu Englisch (8) ist qualitativ anders, es handelt sich um eine bruchstückhafte Übersetzung des Estnischen ins Englische. Das sich erhöhende Sprechtempo deutet jedoch darauf hin, dass die Übersetzung nicht ad hoc verfasst wird, sondern im Voraus vorbereitet wurde. CS 9 (Wechsel zum Russischen) unterscheidet sich durch ein deutlich langsameres Sprechtempo und Pausen, was ein Verweis darauf sein könnte, dass es sich um einen improvisierten Übersetzungsversuch handelt. Darauf deutet auch der spontane Wechsel zum Estnischen (10) hin, dann wieder zum Russischen (11) und anschließend wieder zum Estnischen (12): Nachdem eine Zeile des Liedes auf Englisch rezitiert wurde, verwendet Silver Sepp Estnisch als Relais-Sprache, um die englische Präposition „through“ ins Russische zu verdolmetschen. Das Publikum reagiert auf „läbi“ (est., durch) mit „čerez“ (russ., durch), und Sepp wiederholt den mithilfe des Publikums verdolmetschten Ausdruck auf Russisch. Allerdings endet sein Kommentar mit dem estnischen „jah“.

Situation 3. Während seines Auftritts vermutet Silver Sepp, dass nicht alle Zuschauer den Text des „Regilaul“s verstehen, und fragt auf Russisch ((1); 14:58): „Kak eto perevesti?“ (,Wie übersetzt man das?'). Dann versucht er, den Inhalt auf Englisch wiederzugeben, Zeile für Zeile ((2); 15:05-15:15): „We are searching the thing for ourselves in this world. [sic!] We are cutting the path through the forest for our luck and happiness". Dann unterbricht er seinen Auftritt mit der Frage auf Russisch ((3); 15:16-15:19): „Kak eto po russki, eto?“ (,Wie sagt man das auf Russisch?'), worauf das Publikum in Lachen ausbricht. Als nächstes lädt Silver Sepp eine Zuschauerin auf die Bühne ein. Sie verdolmetscht für ihn eine Zeile des Liedes ins Russische, die Silver Sepp auf Russisch wiederholt (15:20-16:08). Dann wird der Auftritt auf Estnisch fortgesetzt.

Kommentar zu Situation 3. Die Frage (auf Russisch), mit welcher Silver Sepp seinen Auftritt unterbricht, richtet sich direkt an das Publikum und stellt keinen geplanten Teil der Performance dar. CS (1) ist vergleichbar mit CS1 in Situation 2, es ist keine rhetorische Frage, sondern ein Aufruf zum Publikumsdialog. Doch das Publikum reagiert nicht. Die darauffolgende Übersetzung ins Englische scheint ähnlich wie (2) und (4) in Situation 2 bereits 
SHAKHOVA

vorgefertigt zu sein. Der spontane Wechsel zum Russischen (3) hat eine deutlich humoristische Wirkung auf das Publikum. Diese Wirkung hat Silver Sepp offensichtlich erwartet, wovon sein Lächeln und sein Blick in die Richtung der Moderatorin zeugen. Dies scheint ein Beispiel dafür zu sein, was Verschik in Anlehnung an Golovko (1994) als Code Switching-Spiel (,CS game') bezeichnet, nämlich die absichtliche Verwendung von CS zu satirischen Zwecken (Verschik 2005, ${ }^{2}: 382$ ). Die Einladung einer Zuschauerin auf die Bühne stellt eine Fortsetzung des Spiels dar, worauf das Publikum mit Lachen und Applaus reagiert.

Mit CS (1) macht Silver Sepp auf den Bedarf an der Übersetzung aufmerksam, den er als Interpret empfindet. Die Übersetzung hat dabei eine indikative Funktion in multilingualen Diskursen (vgl. formative und indikative Rolle der Übersetzung bei Susam-Sarajeva 2006: 1): Die Übersetzung deutet im beschriebenen Fall auf die Erwartungen der KommunikationsteilnehmerInnen (des sprechenden Subjekts) hin und macht auf die Brüche, Diskrepanzen und metonymischen Lücken im Diskurs (aus der Perspektive des sprechenden Subjekts) aufmerksam. Die formative Funktion der Übersetzung besteht darin, die Rezeption eines Werkes oder Liedes zu beeinflussen.

\section{Schlussfolgerung}

Im Mittelpunkt der vorliegenden Recherche stand der mehrsprachige Diskurs eines internationalen Autorenliedfestivals, welches in Tartu, Estland, jährlich stattfindet und von den VertreterInnen sprachlicher und ethnischer Minderheiten veranstaltet wird.

Die Kommunikationssituationen, die im Rahmen des Festivals „Lehesaju Muusika" entstehen und durch die Machtkonstellation des symbolischen Diskursraums bedingt sind, stellen umfangreiches empirisches Material für Mehrsprachigkeitsuntersuchungen dar. Die im Rahmen der vorliegenden Arbeit durchgeführte mikrosoziologische Falluntersuchung, ein qualitatives Interview sowie Video- und Tonaufnahmeanalysen veranschaulichen die Komplexität dieser Kommunikationssituationen. Sie eignen sich für die Untersuchung der Heteroglossie und Exophonie, für mikrosoziologische Studien der Mehrsprachigkeit von ethnischen Minderheiten in Estland sowie für die Analyse und Interpretation von Code Switching als Element der mehrsprachigen Kommunikation. Betrachtet aus der translationswissenschaftlichen Perspektive, stellen diese Kommunikationssituationen auch ein potenziell facettenreiches Forschungsfeld dar. 
Das internationale Autorenliedfestival „Lehesaju Muusika“ in Tartu

\author{
Anastasia Shakhova \\ ashakhov1988@gmail.com \\ GERMANY
}

\title{
Literatur
}

Auer, P. 1998. From Code-switching via Language Mixing to Fused Lects: Toward a Dynamic Typology of Bilingual Speech. - http://www.inlist.uni-bayreuth.de/ issues/6/inlist6.pdf (30.10.20).

Boss, D. 1985. Das sowjetrussische Autorenlied: eine Untersuchung am Beispiel des Schaffens von Aleksandr Galič, Bulat Okudžava und Vladimir Vysockij. München: Otto Sagner. https://doi.org/10.3726/b12538

Busch, B. 2017. Mehrsprachigkeit. Wien: Facultas.

Even-Zohar, I. 2008. Gesetzmäßigkeiten der kulturellen Interferenz. - M. Krysztofiak, Hrsg., Ästhetik und Kulturwandel in der Übersetzung. Bern: Peter Lang, 117-134. Ins Deutsche übersetzt von K. Lukas.

Gardner-Chloros, P. 2009. Code Switching. New York: Cambridge University Press.

Hammer, S. 2010. Mani Matter und die Liedermacher: zum Begriff des "Liedermachers" und zu Matters Kunst des Autoren-Liedes. Bern: Peter Lang.

Kuhn, T. 1978. Die Struktur wissenschaftlicher Revolutionen. Frankfurt am Main: Suhrkamp Verlag. Ins Deutsche übersetzt von H. Vetter.

Lefevere, A. 2009. Interpretation, Übersetzung, Neuschreibung: Ein alternatives Paradigma. - S. Hagemann, Hrsg., Deskriptive Übersetzungsforschung. Eine Auswahl. Translationswissenschaftliche Bibliothek 4. Berlin: Saxa, 63-92. Ins Deutsche übersetzt von Susanne Hagemann.

Olson, L. 2004. Performing Russia: Folk Revival and Russian Identity. London/New York: Routledge.

Orlova, Y. 2011. Avtorskaja pesnja kak fenomen sovetskoj kultury. - Teorija i praktika obščestvennogo razvitija, 4, 120-123.

Poljakov, F. 2011. Unsere Lieder tragen wir im Herzen von der Wiege an. Bemerkungen zum Massenlied der sowjetischen Epoche. - S.-M. Newerkla, F. Poljakov, O.-J. Schmitt, Hrsg., Das politische Lied in Ost-und Südosteuropa. Münster: LIT Verlag, 113-128.

Population and housing censuses in Estonia, Latvia and Lithuania 2011. https://www. stat.ee/sites/default/files/2020-08/2011\%20Population\%20and\%20Housing\%20 Censuses\%20in\%20Estonia\%2C\%20Latvia\%20and\%20Lithuania.pdf (30.10.20).

Schneider, T. 2013. Demographic Trends in Estonia. Primary results and interpretation. - https://www.kas.de/c/document_library/get_file?uuid=28b94892-9ac5c53f-76ea-6e7b470c2e87\&groupId=252038 (30.10.20).

Susam-Sarajeva, Ş. 2006. Theories on the Move. Translation's role in the Travels of Literary Theories. Amsterdam/New York: Rodopi.

Verschik, A. 2004. Aspects of Russian-Estonian codeswitching: Research perspectives. - International Journal of Bilingualism, 8 (4), 427-448. 
SHAKHOVA

Verschik, A. 2005. The language situation in Estonia. - Journal of Baltic Studies, 36 (3), 283-316. https://doi.org/10.1177/13670069040080040201

Verschik, A. 2005. Multilingualism in Estonia. - Journal of Multilingual and Multicultural Development, 26 (5), 378-390. https://doi.org/10.1080/01629770500000111

Verschik, A. 2016. Language Contact, Language Awareness, and Multilingualism. https://www.academia.edu/30930447/Language_Contact_Language Awareness_and_Multilingualism (30.10.20).

Vihalemm, T., Leppik, M. 2018. Multilingualism and Media-Related Practices of Russian-Speaking Estonians. - S. Lazdina, H.-F. Marten, eds., Multilingualism in the Baltic States. Societal Discourses and Contact Phenomena. Palgrave Macmillan, 238-268. https://doi.org/10.1057/978-1-137-56914-1 